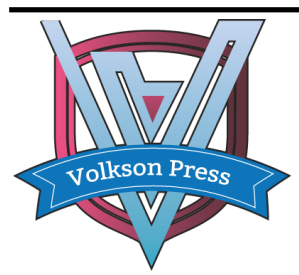

Contents List available at VOLKSON PRESS

Education, Culture and Social Development (ECSD)

DOI : http://doi.org/10.26480/icecsd.01.2018.53.55

Journal Homepage: : https://topicsonsocialdevelop.com/

\title{
A COGNITIVE ANALYSIS OF CHINESE IDIOMS INVOLVING BODY PARTS
}

\author{
Zhong Deng*, Die Hu \\ School of Foreign Languages, Southwest Minzu University, Chengdu, China \\ *Corresponding Author E-mail: icomehere@126.com
}

This is an open access article distributed under the Creative Commons Attribution License, which permits unrestricted use, distribution, and reproduction in any medium, provided the original work is properly cited

\section{ARTICLE DETAILS}

Article History:

Received 12 November 2017 Accepted 12 December 2017 Available online 1 January 2018

\section{ABSTRACT}

In this article we analyze idioms involving body parts in Chinese in an effort to explore their intrinsic formation mechanisms based upon embodied philosophy and perceptual symbol systems. To this end, such idioms are deciphered specifically in the light of their physical features and their situated conceptualization. It is in this process that we find a systematic correspondence between body parts and how they trigger cognitive activities in human mind. This completed, we further list three principles that are in line with the formation of such idioms which are of preliminary but general importance in terms of interpreting how cognitive mechanisms work to incorporate human body into human language.

\section{KEYWORDS}

body parts, embodied philosophy, situated conceptualization, mechanisms.

\section{INTRODUCTION}

Though human beings have long been relied on their body parts to perceive, they seldom yield a conclusion why they finally put their body parts to be the materials in language. On the whole, studies on idioms about body parts are mainly in metaphorical and psychological perspectives. For one thing, idioms about body parts are so universal in many human languages that we can find numerous idioms in different languages with the same underlying meaning. Therefore, comparative studies across language on idioms about body parts extremely prevail, and most of them focus on metaphorical usages that encompass the rhetorical devices of exaggeration and metaphor [1,2]. In another view, focus is given to neural experiments or speculation on animals in facets of subjects such as mental rotation and motion perception $[3,4]$. A sophisticated and delicate part as the brain is, the proceedings in this field seem to be rather intricate yet still conducive. The reason why body parts are involved into language is somewhat different from other objects that also compose parts of a language is that body parts are parts of us that interrelate with neural tissues. Consequently, when discussing the formation of those idioms, it is defective for us to merely talk about metaphors in those idioms. That is why we will focus on the cognitive process in this article based on embodied philosophy and different conceptual systems.

\section{EMBODIED PHILOSOPHY AND PERCEPTUAL SYMBOL SYSTEMS}

\subsection{Embodied Philosophy}

As a group of researchers note that the essence of metaphor is understanding and experiencing one kind of thing in terms of another, the formation of metaphorical language centers on individual perception and experience, of which the idioms about body parts account for a great proportion [5]. Unlike the argument that we tend to picture an object with regards to its features, which weighs on external beings' structure, embodied philosophy sets subjective embodiment as the primordial element for one's perception. Based on interactions with environment, especially perception in the flesh, our bodily conduction can greatly contribute to concepts in our mind [6]. Embodied philosophy has been adopted to explain how language is linked to personal experience. It is believed that language is correlated with people's perceptual system, and people are likely to depict objects based upon their own physical features, which is also called the cognitive activity across registers. At this level, it is a consensus that in most language, metaphors are applied to expressions, among which body parts are a universal tool. In this sense, idioms about body parts are the manifested paradigm of how human experience counts in formulating language.

\subsection{Perceptual Symbol Systems}

In this paper two traits of perceptual symbol systems will be introduced to realize full interpretation. A researcher addressed that perceptual symbols are multi-modal, that is to say, these symbols can be forged from all-around perspectives such as audition, haptics, olfaction and gustation, which all involve some body parts [7]. He also illustrates the cognitive process as two phrases. Firstly, in the perceptual states, when confronting an object, the brain receives signals of an entity from certain possible senses. We can smell it, taste it, lift it, speculate it, or hear it. Then some sensory associates learn to formulate reflections that are part of conscious experience and unconscious neural representations. Then the selective attention would extract from the perceptual states the symbols that can be stored as long-term memory. In the second step, every time we encounter the similar thing, the existing symbols can be activated out of memories by aligning neural associates in terms of sight, hearing, smell and other perceptive vehicles. While the perceptual symbol systems can illustrate the perception of a single body part, a researcher proposed situated conceptualization after his proposal of perceptual symbol systems as supplement, which facilitates illustrating formation process of idioms with body parts in them [8]. In his words, during the activation of memories, concepts are not typically processed in isolation but are typically situated in background settings, events and introspections. That is to say, when referring to a hand, we do not only think about its shape but also think about things it holds, the posture of it and the person it might belongs to. Here we use this theory to intensify the groundings of our statement. Furthermore, it is proved that similar instances can activate similar neural patterns [9].

\section{ANALYSES OF IDIOMS ABOUT BODY PARTS}

\subsection{Body Parts and Cognition}

The seemingly rich vocabulary world always welcomes new expressions, but not all candidates are widely accepted into the noble circle of formal language as those in dictionaries. According to our statistics, there are at least five thousand idioms that contain body parts. Therefore, what it is that lead the body parts into various languages to become a phenomenon is very intriguing. Primarily, the cognition of body parts is special in that every sense of human beings vigorously plays a significant role in their 
perception of the outside world. They are so often applied that it seems that every experience has some bearing on at least a kind of body part. Consequently, we assume everybody part is cognized as a symbolic indicator of feelings or results of conduction correlated with it. For example, we use (yi mai xiang cheng; be intimately tied up with) as an example to explain concretely [10]. Firstly, according to situated conceptualization, in the idiom, the body part, vein, can activate symbols like a line, blood, and piping system. The body part vein can also be connected to the domain of inheritances owing to the fact that it is tied to blood. Based on the notion of family originated from centuries ago, we have the habit of inheritance between kinfolks. As we have tested in medical science, a blood test is one of the most reliable methods to confirm affiliation. When we want to express the meaning of succeeding, our mind can create settings like natural bindings of families, which is blood. The relation of succeeding something is like the continuing existence of one type of blood. As a result, we use the expression to define the intimacy and continuance.

In order to explain the cognitive mechanism of Chinese idioms about body parts, we adopt the models introduced in a researcher's studies. One of the perceptual symbol systems help to explain the process of perception and the formation of a language, in which reality activates the psychology and then are processed into specific language and long-term memory. Jiang, going further, argues that human body structure is tightly tied to how they would shape representation for certain thoughts. That is to say, our body plays a crucial role when we are experiencing things, and that experience counts in the course of perception and conception. This does not only include physical being but also the thoughts produced in active mental parts. In addition, environment also can impact on the perception and neural system. Owing to that view, we argue that language should not only be inspected from social features or the external language structure. By analogy, we assume that man tends to depict linkage between objects based upon their already grasped perception. But there are two questions we would like to address. For one thing, it is worth mentioning that we are concerned with what it is that links mind and language. In other words, what is the specific manipulation in our mind that invites body parts into language? Another thing is whether the body part in an idiom is irreplaceable.

One example is rendered here: (qian chang gua du; to be deeply concerned about). Literally, this idiom means your intestine and abdomen are pulled. But in effect, it indicates a strong feeling of missing the intimate ones or worrying about something. The physical elements here are intestine and abdomen, while the possible environmental element is the social relationships or something precious. As to why intestine and abdomen preponderate other body parts, we would like to clarify in two points. On the one hand, of course, as we all know, intestine and abdomen can never conduct other neural force independently other than their own function, which lies in sense of comfort or hunger. But what is intriguing is that the feeling of missing or worrying resembles the misery of discomfort in abdomen. On the other hand, we assume that it is due to the fact that the state of intestine and abdomen holds an underlying implication of winding. The physical traits of intestine are that it is long and is likely to be used to simulation which will be further explained later. That is to say, the medical insight of body parts' appearance and common sense of their function all contribute to the formation of this idiom. These factors might help to verify abdomen and intestine to preponderate over other bodparts.
Hence, the answers to two questions above are manifest. For the first one the linkage is the likeness between the expected expressive effect and the already grasped perception of human body. Of course, this perception is led by environmental factors. For the second one, it is perceived that the chosen body parts represent the social custom or lasting popular elements in environmental terms, and the likeness between thoughts and body parts caught by neural associates might help one body part to stand out.

\subsection{Body Parts in Idioms}

As to idioms about body parts, we believe that the formulating process is that a specific body part is stored as a symbol featuring its own physical function, color, shape, and situation. For example, a heart is red in the size of a fist with the similar function to a pump. Afterwards, in real life experience, we gradually come to learn that in comparison to other body parts, hearts are more pivotal for its delicate mechanism and correspondence to other organs. Heart diseases are somewhat more fatal in that it is the pump controlling blood supply. Blood is something like electricity. Losing blood means failure of implementing instructions from brains, which culminates in death. And these are all functions of body parts we conclude after years of speculation on ourselves. Therefore, we assume everybody part is a symbolic indicator of feelings or results of conduction related to it. Examples are also given here to clarify the application of situated conceptualization in idioms about body parts. When depicting the sense of losing lives, we use idioms about bodily elements like blood in (xing feng xue yu; a sanguinary slaughter). In the first step, we have in our mind the intention to express the scene of slaughter. According to situated conceptualization, when we want to picture slaughter, we simulate numerous bodies and blood on the ground. As to situated conceptualization of blood, the sensory associates are activated to generate its shape and smell respectively. At this moment, the fluidity of blood simulates rain and smell of it simulates wind, hereby the scene of a slaughter is embedded in this idiom. Another case is (yi shou zhe tian; hide the truth from the masses), in which the hand is overstated to an extend that it can hide the sky. This idiom emphasizes the mightiness or the abuse of power. The reason why it is a hand but not a book or other things is that the hand, signifying power and human will, is the most frequently used parts in fights or other action. And in the case of the idiom (gan dan xiang zhao; be straightforward with each other and share weal and woe) as an example. In medical terms, as we can see from the figure below, the organ liver and gallbladder are closer than neighbors because bile ducts are inlaid inside the liver to transfer gall produced by the liver into the gallbladder. Based on the cognition of this sense, we know that a liver and a gallbladder can connote the meaning of closeness. Thus, all intention of describing closeness could activate the already existed perception, namely, (liver) and (gallbladder). This is how we have created the idiom (gan dan xiang zhao; be straightforward with each other and share weal and woe).

\section{THREE PRINCIPLES IN IDIOMS ABOUT BODY PARTS}

Of all the sampled idioms, we identified three principled trends: body parts that play the role of a container; distance between body parts works as a simulator; body parts referring to a whole image. Our further discussions will proceed based on what is classified and labeled in this table.

\begin{tabular}{|c|c|c|}
\hline Principle & Idioms & Body Part \\
\hline \multirow[t]{5}{*}{$\begin{array}{c}\text { Container } \\
\text { Principle }\end{array}$} & (kou mi fu jian; to praise others with evil hearts) & $\begin{array}{l}\text { Mouth, } \\
\text { Abdomen }\end{array}$ \\
\hline & (yi bi kong chu qi; echo the same opinion) & Nostril \\
\hline & (yi mai xiang cheng; be intimately tied up with) & Vein \\
\hline & (yi shou zhe tian; hide the truth from the masses) & Hand \\
\hline & (man fu jing lun; profoundly informed) & Abdomen \\
\hline \multirow[t]{3}{*}{$\begin{array}{l}\text { Distance } \\
\text { Principle }\end{array}$} & (gu rou xiang lian; as closely linked as flesh and blood) & $\begin{array}{l}\text { Flesh, } \\
\text { Blood }\end{array}$ \\
\hline & (gan dan xiang zhao; be straightforward with each other and share weal and woe) & $\begin{array}{l}\text { Liver, } \\
\text { Gallbladder }\end{array}$ \\
\hline & (cun wang chi han; the cheekbones and the jaws are mutually dependent) & Lip, Tooth \\
\hline \multirow[t]{2}{*}{$\begin{array}{c}\text { Trait } \\
\text { Referent }\end{array}$} & $\begin{array}{l}\text { (kou zhi xin kuai; being frank and outspoken) } \\
\text { (jin kou yu yan; words that like pearls of wisdom) } \\
\text { (ku kou po xin; to persuade patiently earnestly and kindly) }\end{array}$ & Mouth \\
\hline & $\begin{array}{l}\text { (gai tou huan mian; make a complete change) } \\
\text { (pao tou lu mian; expose oneself in public) } \\
\text { (ba mian ling long; be clever in dealing with people) }\end{array}$ & Head, Face \\
\hline
\end{tabular}




\subsection{Container Principle}

As for containers, though the container itself means nothing special besides its biological connotation, it is perceived to be able to activate similar situated conceptualization based on its structure and other features. Some of our body parts indeed play a role of a container or a measurement, for instance, containers with objects are abdomens, stomachs, heads and the forth, and containers with air are noses, trachea, etc. The noteworthy point is that most of the idioms are attached with a quantifier like (yi; one) to specify the extent of something or simply as a container. But in the former case, is the quantifier indispensable? The answer, we assume, is that the container is used to define the extent of a description. The quantifier-used idioms are mostly applied to express the meaning of unity or a sense of extremeness, thus (yi; one) is a normal case in idioms about body parts in Chinese by reason of the rich implication of the Chinese character (yi; one), namely, it means small and also mighty or united. In addition, the seemingly abstract filler is combined with an entity such as the mouth and stomach to depict the situation of a person or the relationship of them. If the filler is correlate with the targeted trait, then is the container an arbitrary choice? For instance, in (kou mi fu jian; to praise others with evil hearts), can the body part mouth and abdomen be replaced by other body parts such as the eyes or the feet? The answer is negative because this idiom is not used to describe someone as sweet or evil, the backbone of this idiom is the body parts. That is to say, we need the trait-related body parts in idioms. Based on the idioms in the table, we can see that the mouth is related to remarks, while the abdomen is linked with mind or thoughts.

\subsection{Distance Principle}

After the emergence of refined medical and scientific studies, human beings learn to map a systematic panorama of their own body parts. Therefore, according to their grasped concept of distance, they can picture the correspondence of one body part to another body parts in terms of distance. There exist in human body many parts that are close in terms of place but differ in functions. In Chinese, we use (liver and gallbladder), (lip and tooth), (bone and flesh) to imply intimacy. In this course, the specific feature of body parts has nothing to do with the relative beings we want to express. But the similarity is that the closeness of A and B are in line with the closeness of two body parts. This is to say, the distance between two body parts can activate the concepts of the intimacy between people. When we want to depict the closeness between two things, our sensory associates might activate same meaning parings as in the case of (be straightforward with each other and share weal and woe), (as closely linked as flesh and blood) and the forth. Therefore, idioms about body parts that interpret relations are likely to be used to in our daily lives.

\subsection{Trait Referent}

Another thing about idioms is that we find that some body parts may have designated meanings. That is to say, when we are applying some body parts to expressions, we might imply them with fixed connotation. According to situated conceptualization, any body part is likely to indicate human beings. But each body part has its own unique perceptual symbols, thus the concepts they may arise must somewhat differ. For instance, (mouth) is usually adopted to present the nature of their speech, as in (kou zhi xin kuai; being frank and outspoken), it means one is frank without subtle mentality. In (jin kou yu yan; pearls of wisdom), it underlies that one's words are trustworthy. In (ku kou po xin; to persuade patiently earnestly and kindly), it refers to that one is making great effort to persuade patiently earnestly and kindly. All of them depend on the body part mouth that represents one's wordings. Idioms about mouth thus can be used to show one's speech manner, credit, and insight, which all show the characteristics of the people. In much the same way, in the case of (gai tou huan mian; make a complete change), (pao tou lu mian; expose oneself in public) and (ba mian ling long; be clever in dealing with people), the body part face is adopted to represent personal image.

\section{CONCLUSIONS}

Through the embodied philosophy and perceptual symbol system, we have analyzed the likelihood of their entry into the idioms and exactly how body parts are embedded into idioms. After that, we introduce three types of idioms about body parts according to their semantic structure. It is intriguing that there are principles which help to distinguish one idiom from another. As perceptual symbols and also sense-perception vehicles, body parts in language is a vital phenomenon. It is inspiring how body parts are used as embodiment of emotion or relations independent from their biological functions. In fact, studies about body parts are prevailing but the in-depth analyses of their cognitive process are rare. This article is dedicated to raising awareness of further studies on Chinese idioms whose morphologic patterns are distinctive, thus assisting in the construal and translation of Chinese idioms about body parts.

\section{REFERENCES}

[1] Yang, Z.Q. 2010. A Study on the Metaphor About Body Parts in French and Chinese Expressions. Guangdong, Sun Yat-Sen University.

[2] Chen, C.Y. 2017. Comparative Study of English and Chinese Body-Part Idioms Based on Conceptual Metonymy. Chengdu, Southwest Jiaotong University.

[3] Anne Bo"ckler, Günther Knoblich, Natalie Sebanz. 2011. Giving a Helping Hand: Effects of Joint Attention on Mental Rotation. Experimental Brain Research, 211, 531-545.

[4] Schouten, B., F. Troje, N., Verfaillie, K. 2011. The Facing Bias in Biological Motion Perception: Structure, Kinematics, and Body Parts. Attention, Perception Psychophysics, 73, 130-143.

[5] Lakoff and Johnson. 1980. Metaphors We Live By. Chicago, University of Chicago Press.

[6] Lakoff and Johnson. 1999. Philosophy in the Flesh: The Embodied Mind and Challenge to Western Thought. New York, Basic Books.

[7] Barsalou, L.W. 1999. Perceptual Symbol System. Behavioral and Brain Science, 22, 577-660.

[8] Barsalou, L.W. 2009. Simulation, Situated Conceptualization, and Prediction. Philosophical Transactions of the Royal Society. Philosophical Transactions of the Royal Society B, 364, 1281-1289.

[9] Cree, G.S., McRae, K. 2003. Analyzing the Factors Underlying the Structure and Computation of the Meaning of Chipmunk, Cherry, Chisel, Cheese, and Cello. Journal of Experimental Psychology: General, 132 (2), 163-201.

[10] Jiang, M. 2014. A Probe into the Embodiment Hypothesis in Cognitive linguistics. Foreign Language and Literature, 2, 55-59.

[11] John, R.S. 1980. Minds, brains and programs. Behavioral and Brain Science, 3, 417-457. 